\title{
Cross-modal transfer of stimulus control in the albino rat: A stimulus delay procedure
}

\author{
JAMES S. MacDONALL and HENRY MARCUCELLA \\ Boston University, Boston, Massachusetts 02215
}

\begin{abstract}
The present experiment demonstrated in a simultaneous discrete trial discrimination that the stimulus control of a rat's leverpress response can be errorlessly transferred across stimulus modalities, i.e., from light to click location and from click to light location. Subsequent to acquisition of the original discrimination, the original and new discriminative stimuli were simultaneously presented for several sessions. Then the new discriminative stimulus was presented 3 sec prior to the onset of the original discriminative stimulus. Within the direction of transfer, e.g., from light to click location, the delay group emitted fewer trial and intertrial errors than the control group. As the new discriminative stimuli acquired control over responding, the response latency distributions were differentially affected. The results suggest that the transfer of control from the original to the new discriminative stimuli is mediated by the temporal aspects of the delay interval.
\end{abstract}

The stimulus control exerted by a particular set of stimuli can be errorlessly transferred to a new set of stimuli by (a) superimposing new stimuli on the stimuli exerting control and then (b) gradually eliminating the discriminative properties of the initial stimuli (stimulus fading procedure). Recently, Touchette (1971) presented a variation of the fading procedure in which the physical properties of the stimuli remain unchanged but the time between the onset of the new and original stimuli was gradually increased contingent upon the occurrence of a correct response (stimulus delay procedure). On the first trial, both sets of stimuli were simultaneously presented. A correct response resulted in a $1 / 2-\mathrm{sec}$ delay between the onset of each of the stimuli on the next trial, i.e., on the next trial, the delay between the onset of the new discriminative stimuli and the original discriminative stimuli equaled $1 / 2 \mathrm{sec}$. A correct response on this and each succeeding trial further increased the delay by $1 / 2 \mathrm{sec}$. If an incorrect response occurred on any trial, the duration of the delay was decreased by $1 / 2 \mathrm{sec}$ on the subsequent trial. The use of such a gradually increasing delay not only resulted in the errorless transfer of stimulus control, but also allowed the specification of the exact point in time at which transfer occurred.

The results of Touchette have been extended to animal subjects by Brown and Rilling (1975). Using pigeons and a gradually increasing delay procedure, Brown and Rilling were able to transfer stimulus control from a horizontal-vertical discrimination to a red-green discrimination. In addition, they demonstrated that Touchette's procedure facilitated

This research was supported in part by Grant 381-PS from Boston University Graduate School to the second author. Reprints may be obtained from James S. MacDonall, Washingtonian Center for Addictions, 41 Morton Street, Boston, Massachusetts 02130. the transfer of stimulus control, and thus did not function.solely as a measuring technique.

In both the stimulus fading and stimulus delay procedures, the new and original stimuli are first simultaneously presented. However, the stimulus delay procedure differs from the stimulus fading procedure in that the physical characteristics of the stimuli remain unchanged. The effect of this difference is that transfer of control is not forced by the experimenter as it is with the stimulus fading procedure. Because the physical properties remain constant, it is possible for the subject to simply wait for the onset of the original discriminative stimulus. One interesting question, then, is why transfer should occur at all with this procedure. One possible interpretation is that as the delay between the onset of the stimuli gradually increases, the overall reinforcement frequency decreases. Perhaps this decrease in reinforcement frequency increases the probability of responding in the presence of the new discriminative stimulus (Bloomfield, 1967; Terrace, 1966).

A second possibility is that the temporal aspects of the situation are the important determiners of transfer. Is it the introduction of a temporal relationships between the stimuli, per se, rather than the resulting change in reinforcement frequency that facilitates transfer? If temporal variables are important, then transfer should occur if a fixed, rather than gradually increasing, delay is imposed between the onset of the stimuli. In addition, transfer should occur even if the effect of the delay interval on reinforcement frequency is minimal. The present experiment examined these possibilities. A betweensubjects design was employed. Secondly, the present study extended the results of Brown and Rilling (1975) and Touchette (1971) by demonstrating that the stimulus delay procedure was effective in trans- 
"erring stimulus control of a rat's leverpress across stimulus modalities.

It is important to note that, even in situations where stimulus control is well established, the disruptive effects of introducing a novel stimulus are considerable (Brown \& Rilling, 1975). To eliminate such effects, the delay between the onset of the new and original discriminative stimulus was introduced only after several sessions during which the stimuli were simultaneously presented. Control animals were also exposed to this simultaneous pairing procedure.

In order to determine if the results were dependent upon the particular direction of the transfer of stimulus control, the control was transferred, in different groups of animals, from light location to click location, and vice versa.

Finally, to facilitate cross-modal transfer, stimuli were needed that would be readily discriminable within each modality, yet share a common element across modalities. The locations of auditory and visual stimuli met both requirements. Click and light stimuli located adjacent to each other would share the common element of location, yet discriminability could be maximized within each modality by locating the stimuli at opposite ends of the chamber. Similarly, response mechanisms were located at opposite ends of the chamber to minimize response generalization.

\section{METHOD}

\begin{abstract}
Subjects
Eight Sprague-Dawley male albino rats, 90 to 150 days old, were maintained at $85 \%$ of their free-feeding weights. Throughout the experiment, water was avilable only in the home cage. Rats $R 1$, R2 and R3 had been previously exposed to variations of the basic discrimination schedule, employing a click location as the discriminative stimulus (see below). Rats R4, R5, R6, R7, and R8 were experimentally naive.
\end{abstract}

\begin{abstract}
Apparatus
The experimental chamber was a modified Gerbrands conditioning unit, 7.8 in. $(20.8 \mathrm{~cm}) \times 14.9$ in. $(37.8 \mathrm{~cm}) \times 7.5 \mathrm{in}$. $(19.0 \mathrm{~cm})$ in height. Mounted on the walls at each end of the chamber were (1) a standard Gerbrands rat lever, (2) a food magazine, and (3) a stimulus lamp above and to the left of the lever. A 1 -in. $(2.54-\mathrm{cm})$ diameter opening was cut into each end wall of the chamber above and to the right of the lever and covered with wire mesh. Two $24-V$ heavy-duty relays, suspended from the ceiling of the sound-attenuating chest enclosing the chamber, were located $1 \mathrm{in} .(2.54 \mathrm{~cm})$ behind the mesh-covered openings but not in contact with the chamber. Each relay was activated by a Grason-Stadler timer to operate 10 times/sec. The locations of the illuminated lamp or of the clicking relay were the discriminative stimuli in the experiment.

Forty-five-milligram Noyes food pellets delivered by pellet dispensers located behind each lever were used as reinforcers. Standard electromechanical programming equipment used to control the presentation of the stimuli and to record the data were located in an adjacent room.
\end{abstract}

\section{Procedure}

The discriminative stimuli were presented automatically in a discrete trials procedure. A trial was defined as the period of tume durng which any of the discriminative stimuli were present All trials were terminated by the first response on elther lever. A correct response was defined as a response on the lever adjacent to the discriminatıve stımulus presented. That is, a response on the left lever was correct when the left lamp was illuminated or when the left relay was clickıng. Simılarly, a response on the right lever was correct when the right lamp was illuminated or when the right relay was clicking. All correct responses were reinforced. A trial error was recorded when a response occurred on the lever not adjacent to the discriminative stimulus. An intertrial error was recorded when a response occurred and no discrıminative stımulus was present.

The scheduled duration of the intertrial periods was, on the average, $120 \mathrm{sec}$ and was determined by the following sequence of intervals: $25,55,10,295,105,145,225,120,21,130,75,335$, 132,193 , and $50 \mathrm{sec}$ To eliminate intertrial responding, the onset of a trial was determined by a procedure similar to that of Marcucella (1974). A trial was started when one of. the aforementioned intertrial intervals had elapsed and no response had occurred. A response on either lever during an intertrial period lengthened the intertrial period by a duration equal to the next scheduled interval. The timing of the additional interval began when the timing of the current interval had elapsed. The leftrıght order of stimulus presentation followed a predetermined semirandom sequence. Sessions were terminated after the delivery of 100 reinforcers. The houselight was on during the session.

Click-location discrimination training. R4 was first magazine trained to each food magazine in the presence of the adjacent click stimulus. Leverpress shaping began in the following session, again in the presence of the adjacent stimulus. After the first 15 responses on each lever were reinforced, the discrete trials procedure began. Since R1, R2, and R3 were already leverpressing, they were immediately placed on the discrete trial, procedure The click-location discrimination continued until the criterion of fewer than 2 intertrial errors $/ \mathrm{h}(0.033$ responses $/ \mathrm{min})$ over five sessions was reached. For purposes of calculating the criterion, responses occurring within $1 \mathrm{sec}$ of a trial response (either correct or error) were omitted, since Blough (1963) has suggested that these responses are under the control of the prior response and not under the control of a stımulus in the external environment. After reaching the criterion, a click location stimulus and its adjacent light location stimulus were stimultaneously presented on each trial for $3 \frac{1 / 2}{2}$ sessions (see Table 1 for a summary of the procedure).

Light Jocation discrimination training. Rats R5, R6, R7, and R8 were magazine trained and leverpress trained in the manner of R4, except the discriminative stimuli were light locations. The light-location discrimination continued until the same criterion was reached. Then a light-location stimulus and its adjacent click-location stimulus were simultaneously presented on each trial for $31 \frac{1}{2}$ sessions.

Abrupt procedure. For rats R2, R3, R5, R7, and R8, the initial discriminative stimuli were removed from the experiment after

Table 1

Sequence of Procedures for Abrupt and Delay Groups

\begin{tabular}{|c|c|c|c|c|}
\hline \multirow[b]{2}{*}{ Sessions } & \multicolumn{2}{|c|}{ Abrupt Group } & \multicolumn{2}{|c|}{ Delay Group } \\
\hline & $\mathrm{R} 5, \mathrm{R} 6, \mathrm{R} 7 *$ & $\mathrm{R} 2, \mathrm{R} 3$ & $\mathrm{R} 7, \mathrm{R} 8$ & R1, R4 \\
\hline To Criterion & light & click & light & click \\
\hline 3.5 & $\begin{array}{l}\text { light and } \\
\text { click }\end{array}$ & $\begin{array}{l}\text { light and } \\
\text { click }\end{array}$ & $\begin{array}{l}\text { light and } \\
\text { click }\end{array}$ & $\begin{array}{l}\text { light and } \\
\text { click }\end{array}$ \\
\hline 10 & click & light & $\begin{array}{l}\text { click** } \\
\text { light }\end{array}$ & $\begin{array}{l}\text { light*** } \\
\text { click }\end{array}$ \\
\hline To Criterion & click & light & click & light \\
\hline
\end{tabular}

${ }^{*} R 7$ changed to the delay group after three sessions of the click-location discrimination.

**3 sec delay 
$31 / 2$ sessions of simultaneous presentation. Thus, rats R2 and R3 continued to have a light-location stimulus presented on each trial, and rats $\mathrm{R} 5, \mathrm{R} 7$, and $\mathrm{R} 8$ a click location stimulus. With the exception of $R 7$, training continued until the criterion was again met. The click-location stimuli apparently failed to acquire control over the responses of $\mathrm{R} 7$; that is, $\mathrm{R} 7$ ceased responding. The following session began with light locations as the discriminative stimuli. After 11 trials, light was removed and the click stimuli were presented. Again, R7 failed to respond and the session was terminated. On the third day, light locations were again the discriminative stimuli for the first 11 trials. $\mathrm{R} 7$ again failed to respond when the click locations were the discriminative stimuli. At this point, the trial was terminated by the experimenter and the delay procedure was employed for the remainder of this and the following sessions (see below).

Delay procedure. After rats $\mathrm{R} 1, \mathrm{R} 4$, and $\mathrm{R} 8$ were exposed to $3 \mathrm{t} / 2$ sessions of simultaneous stimulus presentation, a 3-sec delay was imposed between the onset of the new discriminative stımulus and the old discriminative stimulus which followed. For rats R1, and R4, the light location stımulus preceded by $3 \mathrm{sec}$ the onset of the click-location stimulus. A response on either lever during the first $3 \mathrm{sec}$ of the light-location stimulus terminated the stimulus and prevented the onset of the click-location stimulus on that trial. If no response occurred for $3 \mathrm{sec}$, then the click-location stımulus was also presented. Both stimuli were continued until the rat responded. After 10 sessions, the click-location stımuli were removed from the experiment. The light-location discriminatıon continued until the criterion was met.

For rats R7 and R8, the light-location stimuli preceded by $3 \mathrm{sec}$ the onset of the click-location stimuli. A response on either lever during the first $3 \mathrm{sec}$ of the click-location stimulus terminated the stimulus and prevented the onset of the light-location stimulus on that trial. If no response occurred for $3 \mathrm{sec}$, then the light location stimulus was presented. Both stimuli continued until the rat responded. After 10 sessions, the light-location stimulus was removed from the experıment. The click-location discrımınation continued until the criterion was met.

Due to an experimenter's error, rats R5 through R8 were exposed to 3 rather than $3 \frac{1}{2}$ sessions of simultaneous stimulus presentation.

\section{RESULTS}

\section{Click-Location and Light-Location Discrimination}

All subjects met the criterion. An analysis of variance of trial and intertrial errors occurring in the last five sessions prior to the simultaneous stimulus presentation revealed no significant differences between the four groups [F/trial errors $(3,4)=5.44 ; \mathrm{p}>.05, \mathrm{~F} /$ intertrial $\operatorname{errors}(3,4)=$ $: 45, \mathrm{p}>.05]$.

\section{Performance During the Transfer of Stimulus Control}

The total number of trial errors emitted by each subject during the first five sessions of the transfer procedure (delay or abrupt) is shown in Table 2.

Stimulus control transferred from the original to the new discriminative stimuli for both delay and abrupt animals. However, as can be seen from the data of Table 2, the delay procedure produced fewer trial errors than the abrupt procedure regardless of the direction of transfer. Unfortunately, the zero variance for the delay group that was transferred
Table 2

The Number of Trial Errors and Intertrial Errors* During the First Five Sessions Following the Introduction of the Delay or Abrupt Transfer Procedures

\begin{tabular}{lcr}
\hline $\begin{array}{l}\text { Durection of } \\
\text { Transfer }\end{array}$ & Delay & Abrupt \\
\hline Click to & $0(17)$ & $2(143)$ \\
Light & $0(29)$ & $6(103)$ \\
Light to & $11(46)$ & $26(130)$ \\
Click & $12(58)$ & $34(112)$ \\
\hline
\end{tabular}

*Intertrial errors are in parentheses.

to light-location prohibits the use of parametric statistics to compare that group with any other group. Table 2 also shows that fewer trial errors occur during transfer to light location than to click location.

When stimulus control was transferred to click location, the delay procedure produced significantly fewer trial errors than the abrupt procedure, $t(2)=$ $4.59, \mathrm{p}<.025$. Within the abrupt procedure, significantly fewer trial errors occurred when control was transferred to light location, $\mathrm{t}(2)=5.813, \mathrm{p}<.025$. When stimulus control was transferred to light location, the delay procedure again produced fewer trial errors. Within the delay procedure, fewer trial errors occurred when control was transferred to light location. Although it was not possible to test the statistical significance of the last two comparisons, these differences are consistent with the results of the first two comparisons.

Table 2 also shows the total number of intertrial errors (in parenthesis) emitted by each rat during the first five sessions of the transfer procedure (delay or abrupt). The distribution of intertrial errors is similar to the distribution of trial errors. The abrupt procedure produced more errors than the delay procedure; the transfer to click location produced more errors than the transfer to light location. An analysis of variance revealed a significant difference only for the transfer procedure $[F(1,4)=51.65, p<.005]$.

\section{R7}

A detailed analysis of the data of $\mathrm{R} 7$ is of interest for two reasons. First, $\mathrm{R} 7$ failed to transfer in the abrupt procedure and did transfer when the delay procedure was instituted. Second, the behavior of R7 in the delay procedure is typical of the behavior of other subjects in the delay groups.

On the first trial of the abrupt procedure with the click location as the discriminative stimuli, R7 emitted a correct response. This was followed by a trial error on each of the following nine consecutive trials. On the 10th trial, R7 failed to respond, and the trial and the session were terminated after $2 \mathrm{~h}$. The next day, $R 7$ failed to respond on the first trial with the click location as the discriminative stimuli. Again, the session was terminated after $2 \mathrm{~h}$ had 
elapsed on the first trial. On the third day, the trial was terminated after $60 \mathrm{sec}$ of the click-location stimulus. The delay procedure was then instituted.

Figure 1 shows the distribution of correct response latencies of left and right lever responses for $\mathrm{R} 7$ (delay procedure) and R6 (abrupt procedure). Both animals were transferred from light to click location. Latency data are provided for the last session of baseline and simultaneous stages and for the first five sessions of the transfer procedure. Latencies are grouped into 1-sec class intervals with all latencies $\geqslant 6 \mathrm{sec}$ included in the seventh class interval. The

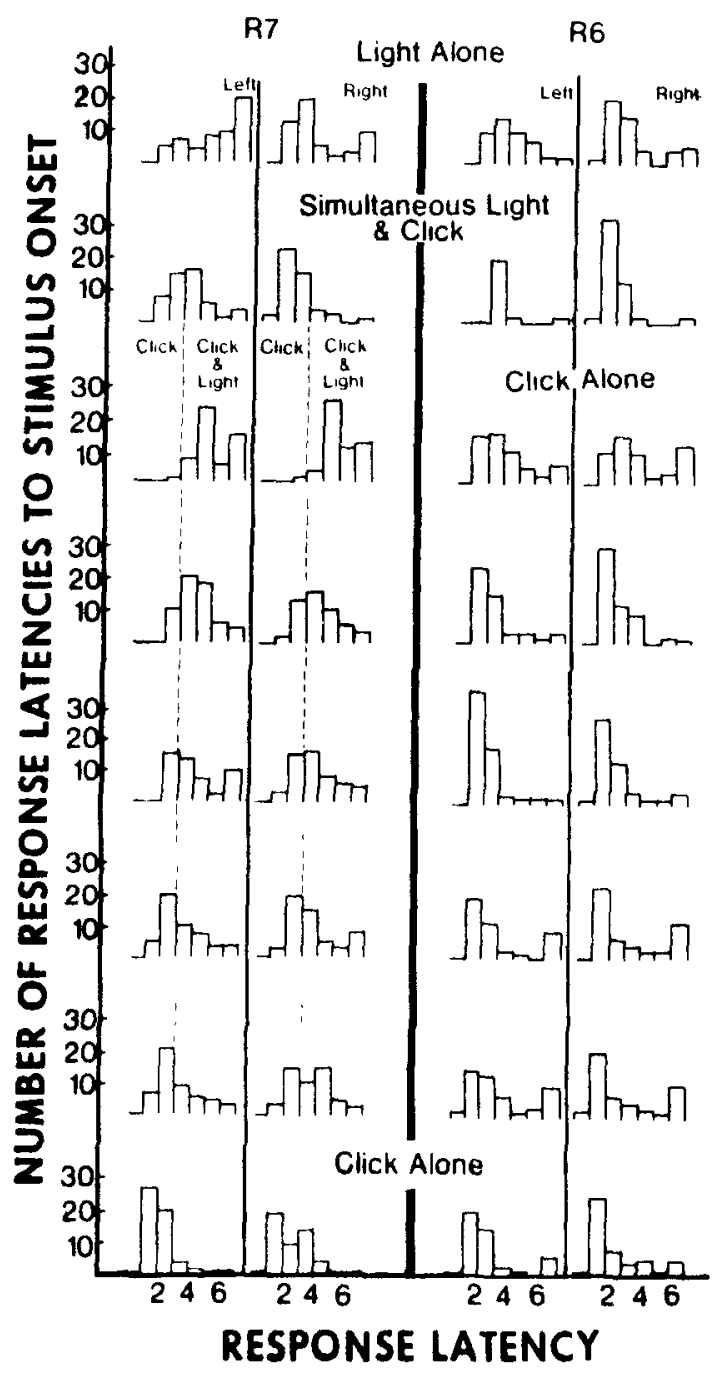

lone sec. intervals)

Figure 1. Correct response latency distributions for left- and right-lever responses for the last session of baseline (light location), the last session of simultaneous presentation of light and click location, the first five sessions of the Iransfer procedure, and the first session of click location alone. Latencies are grouped into 1-sec class intervals with all latencies $\geqslant 6 \mathrm{sec}$ included in the seventh class interval. For the sake of clarity, latency distributions are presented only for $\mathbf{R 6}$ of the abrupt group and $\mathrm{R} 7$ of the dełay group. dashed lines in the left half of the figure represents the time at which the click location stimulus was presented.

Figure 1 shows that $R 7$ (left half) transferred to the click-location dimension by the fifth transfer session. However, there is no exact point of transfer. Instead, there is a gradual increase in the number of responses occurring during the delay interval. During the first transfer day, several responses occurred in the presence of the click alone, the frequency of latencies less than $3 \mathrm{sec}$ increased during the next 3 days until the modal latency was less than $3 \mathrm{sec}$. These results are typical of all animals in the delay groups.

The effect of abruptly removing the original discriminative stimuli (R6) was to (1) decrease the degree of kurtosis of the latency distributions and (2) increase the number of latencies occurring in the last class interval $(\geqslant 6 \mathrm{sec})$. That is, following the abrupt removal of the light location stimuli, R6 did not immediately respond to the onset of the clicklocation stimuli. In fact, several of these latencies were of extremely long duration (for one animal in the abrupt group they reached a maximum of $7,000 \mathrm{sec}$ ). However, when a response was emitted, it occurred on the lever adjacent to the clicking stimulus and was recorded as a correct response.

\section{DISCUSSION}

The results of the present experiment extend those of Brown and Rilling (1975) and Touchette (1971) to a new species and to stimuli in different modalities. In addition, the present results demonstrate that a gradually increasing stimulus delay is not essential to the effectiveness of the procedure. A stimulus delay of fixed duration also facilitates transfer without changing the overall frequency of reinforcement. However, the use of a constant 3-sec-delay interval resulted in the progressive transfer of stimulus control rather than the sudden transfer that was reported by both Brown and Rilling (1975) and Touchette (1971). The reasons for this difference are not clear at the present time.

The present results also suggest a possible advantage of stimulus-delay procedures. The occurrence of transfer does not seem to depend on whether the new stimulus acquired at least some control over responding during the simultaneous pairing of the new and original and discriminative stimuli (Schusterman, 1966, 1967). For example, following the simultaneous pairing of the stimuli, the new discriminative stimulus failed to acquire any control over the responding of $R 7$. When $R 7$ was placed in the delay condition, responding was reestablished and stimulus control was transferred to the new discriminative stimulus. That the new discriminative stimulus failed to acquire any control following simultaneous presentation is not surprising. Reynolds (1961) 
has demonstrated that reinforcement in the presence of simultaneously presented stimuli does not guarantee that both stimuli will acquire control over the response.

The systematic change in the response latencies for the animals in the delay groups suggests that the transfer of control was a two-stage process. During Stage 1, temporal aspects of the delay interval acquired control over responding. A fixed 3-sec delay interval provides the rat with an additional cue, i.e., a temporal cue, to the onset of the old discriminative stimulus. Marcucella (1974) demonstrated that rats temporally discriminate reinforcer availability on DRL schedules less than $30 \mathrm{sec}$ even though reinforcer availability was signaled by auditory cues. In the present experiment, if the onset of the old discriminative stimulus is also temporally discriminated, then one would predict responding to occur near the end of the delay interval. In Stage 2, the new discriminative stimuli acquired control over responding. Once responses near the end of the delay interval occur and are reinforced, they will recur. Shorter response latencies will then be reinforced until most responses occur at the beginning of the delay interval.

Both Brown and Rilling (1975) and Touchette (1971) reported observations consistent with the present two-stage analysis. Brown and Rilling observed their pigeons oriented in front of the new discriminative stimulus during the delay interval and making aborted pecks toward the key before actually pecking the key during the delay intervals. Similarly, Touchette reported that following several trials of the increasing delay, each subject would place his hand on, but not press, the correct response mechanism when the new discriminative stimulus appeared. As soon as the initial discriminative stimulus appeared, the subject would then press the mechanism. Following several trials during which this behavior occurred, the subject pressed immediately after the new discriminative stimulus appeared.

The temporary increase in the frequency of intertrial errors occurring immediately after the introduction of the abrupt transfer procedure has not been reported by other investigators. The reasons are numerous. In many cases, an intertrial response was not possible. For example, Schusterman (1966, 1967) removed the manipulanda during the intertrial interval, while Brown and Rilling (1975) imposed a blackout condition. In other cases, intertrial response data was not reported (Terrace, 1963b). There is evidence, however, that the abrupt introduction of a new discriminative stimulus during acquisition results in an increase in frequency of intertrial responding. Terrace (1963a) reported increases in intertrial responding, when the negative stimulus $(\mathrm{S}-)$ was introduced abruptly, at full intensity and duration, either after only a few (early constant) or after many (late constant) pretraining trials to the positive stimulus $(\mathrm{S}+)$ alone. Although there are many differences between the experiments (acquisition vs. transfer, successive vs. simultaneous discrimination, and pigeons vs. rats), Terrace's data support the findings of the present study that stable low rates of intertrial responding can be significantly increased by abruptly introducing a new discriminative stimulus.

The two-stage analysis presented here suggests a possible explanation of the differential occurrence of intertrial responding in the present experiment. Throughout the delay procedure, the variables controlling the rats' responding were, in turn, con'trolled by the experimenter. Initially the original discriminative stimulus, then the temporal aspect of the delay, and finally the new discriminative stimulus controlled responding. Since these variables were controlled by the experimenter, responding in the intertrial period remained at a low rate. The abrupt procedure did not allow the experimenter to maintain control over responding. When the old discriminative stimulus was abruptly removed from the experiment, the new discriminative stimulus exerted weak control over responding. This is evidenced by both the flattened latency distributions and the rightward shift of the modal latency of R6 (Figure 1, Row 3). Responses terminating these long latencies were under the control of some stimulus not controlled or specified by the experimenters. Since this stimulus might also be present during the intertrial interval and responses terminating these long latencies were reinforced, the control of leverpressing by this other stimulus was strengthened. Thus, the occurrence of this uncontrolled stimulus during the intertrial interval would set the occasion for responding.

\section{REFERENCES}

Bloomfield, T. M. Behavioral contrast and relative reinforcement frequency in two multiple schedules. Journal of the Experimental Analysis of Behavior, 1967, 10, 151-158.

Blough, D. Interresponse time as a function of continuous variables: A new method and some data. Journal of the Experimental Analysis of Behavior, 1963, 6, 237-246.

Brown, C. H., \& Rilling, M. E. Stimulus delay and the reduction of errors in the transfer of stimulus control. Animal Learning \& Behavior, 1975, 3, 21-27.

Marcucella, H. Signalled reinforcement in differentialreinforcement-of-low-rate schedules. Journal of the Experimental Analysis of Behavior, 1974, 22, 381-390.

REYNolds, G. S. Attention in the pigeon. Journal of the Experimental Analysis of Behavior, 1961, 4, 203-208.

SChUSTERman, $R$. Serial discrimination-reversal learning with and without errors by the California sea lion. Journal of the Experimental A nalysis of Behavior, 1966, 9, 593-600.

Schusterman, R. Attention shift and errorless reversal learning by the California sea lion. Science, 1967, 156, 833-835.

Terrace, H. S. Discrimination learning with and without "errors." Journal of the Experimental Analysis of Behavior. 
$1963,6,1-27$ (a)

TERRACE, H. S. Errorless transfer of a discrimination across two continua. Journal of the Experimental Analysis of Behavior. 1963. 6. 223-232. (b)

TerRace, H. S. Stimulus control. In W. K. Honig (Ed.), Operant behavior: Areas of research and application. New York: Appleton-Century-Crofts, 1966.
Touchette. P. Transfer of stimulus control: Measuring the moment of transter Journal of the Expermental Analises of Behavior, 1971, 15, 347-354.

(Received for publication December 31, 1974; revision accepted December 16,1975 .) 